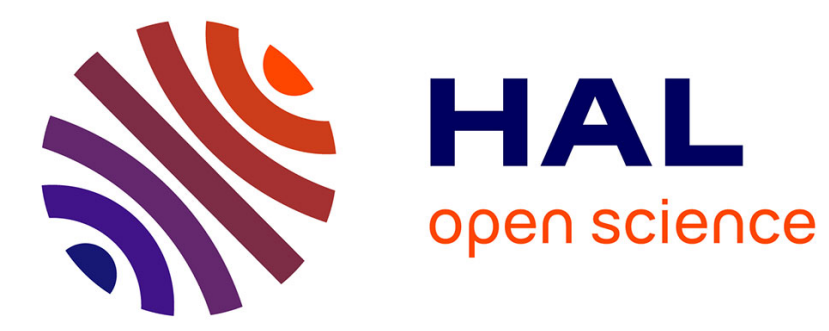

\title{
Fluid trapping during capillary displacement in fractures
}

\author{
Zhibing Yang, Insa Neuweiler, Yves Méheust, Fritjof Fagerlund, Auli Niemi
}

\section{To cite this version:}

Zhibing Yang, Insa Neuweiler, Yves Méheust, Fritjof Fagerlund, Auli Niemi. Fluid trapping during capillary displacement in fractures. Advances in Water Resources, 2016, 95, pp.264-275. 10.1016/j.advwatres.2015.07.015 . insu-01185349

\section{HAL Id: insu-01185349 \\ https://hal-insu.archives-ouvertes.fr/insu-01185349}

Submitted on 20 Aug 2015

HAL is a multi-disciplinary open access archive for the deposit and dissemination of scientific research documents, whether they are published or not. The documents may come from teaching and research institutions in France or abroad, or from public or private research centers.
L'archive ouverte pluridisciplinaire HAL, est destinée au dépôt et à la diffusion de documents scientifiques de niveau recherche, publiés ou non, émanant des établissements d'enseignement et de recherche français ou étrangers, des laboratoires publics ou privés. 


\section{Accepted Manuscript}

Fluid trapping during capillary displacement in fractures

Zhibing Yang, Insa Neuweiler, Yves Méheust, Fritjof Fagerlund, Auli Niemi

PII:

DOI:

Reference:

To appear in:

Received date:

Revised date:

Accepted date:
S0309-1708(15)00162-1

10.1016/j.advwatres.2015.07.015

ADWR 2427

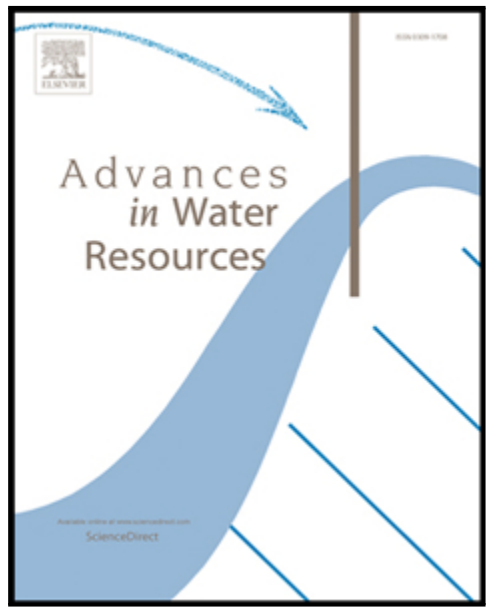

Please cite this article as: Zhibing Yang, Insa Neuweiler, Yves Méheust, Fritjof Fagerlund, Auli Niemi , Fluid trapping during capillary displacement in fractures, Advances in Water Resources (2015), doi: 10.1016/j.advwatres.2015.07.015

This is a PDF file of an unedited manuscript that has been accepted for publication. As a service to our customers we are providing this early version of the manuscript. The manuscript will undergo copyediting, typesetting, and review of the resulting proof before it is published in its final form. Please note that during the production process errors may be discovered which could affect the content, and all legal disclaimers that apply to the journal pertain. 
Highlights

We elucidate how aperture field statistics affects fluid trapping in a fracture

We examine the role of in-plane curvature on fluid displacement and trapping

We present a quantitative analysis of the size distribution of trapped fluid clusters

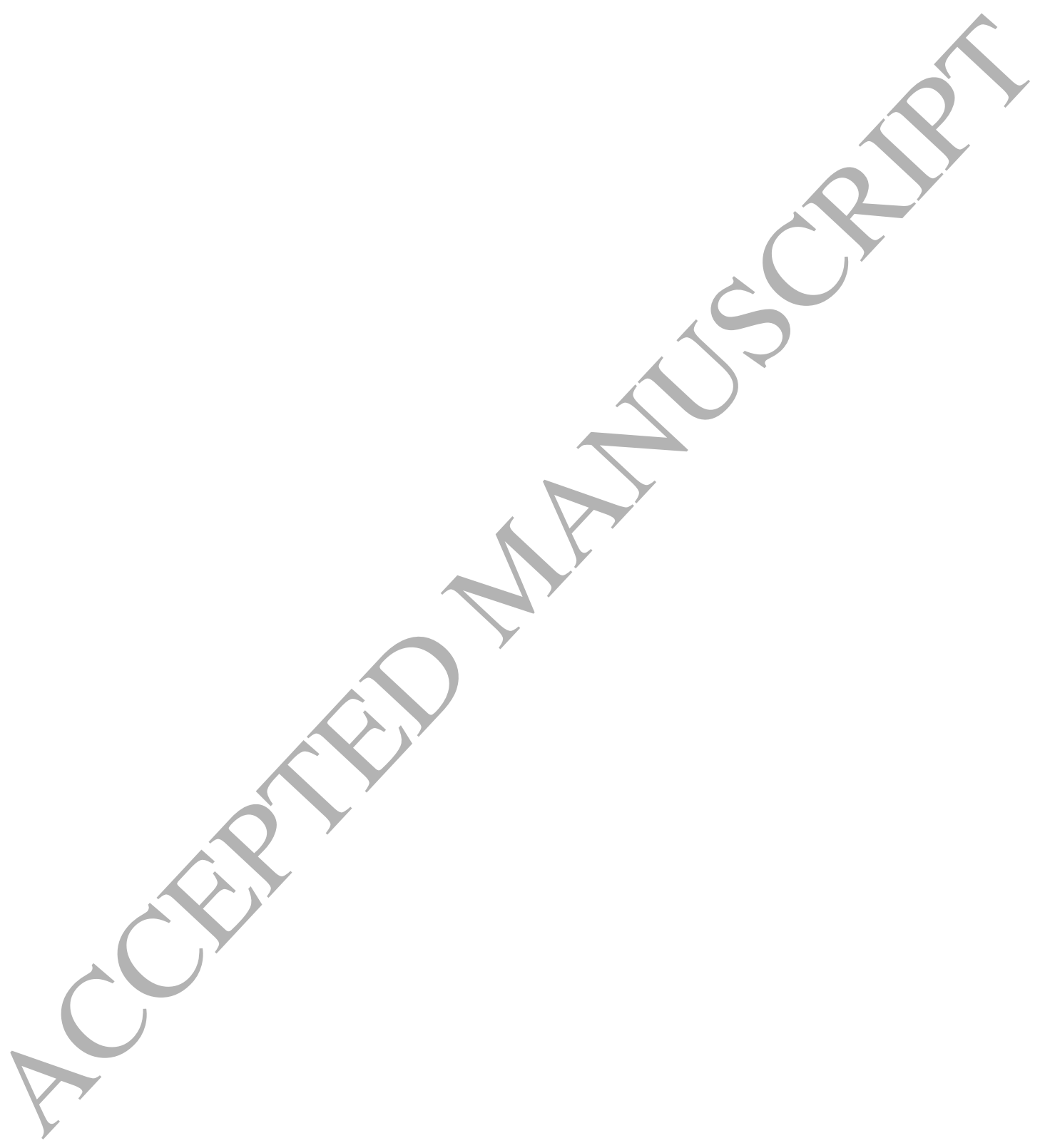




\section{Fluid trapping during capillary displacement in fractures}

Zhibing Yang ${ }^{1,2}$, Insa Neuweiler ${ }^{3}$, Yves Méheust ${ }^{4}$, Fritjof Fagerlund ${ }^{1}$, Auli Niemi $^{1}$

1. Department of Earth Sciences, Uppsala University, Uppsala, Sweden

2. now at Department of Civil and Environmental Engineering, Massachusetts Institute of Technology, Cambridge, Massachusetts, USA

3. Institute of Fluid Mechanics and Environmental Physics in Civil

Engineering, Leibniz University Hannover, Germany

4. Université de Rennes 1, Geosciences Géosciences Rennes (UMR/CNRS 6118), Rennes, France

Abstract. The spatial distribution of fluid phases and the geometry of fluid-fluid interfaces resulting from immiscible displacement in fractures cast decisive influence on a range of macroscopic flow parameters. Most importantly, these are the relative permeabilities of the fluids as well as the macroscopic irreducible saturations. They also influence parameters for component (solute) transport, as it usually occurs through one of the fluid phase only. Here, we present a numerical investigation on the critical role of aperture variation and spatial correlation on fluid trapping and the morphology of fluid phase distributions in a geological fracture. We consider drainage in the capillary dominated regime. The correlation scale, that is, the scale over which the two facing fracture walls are matched, varies among the investigated geometries between $L / 256$ and $L$ (self-affine fields), $L$ being the domain/fracture length. The aperture variability is quantified by the coefficient of variation $(\delta)$, ranging among the various geometries from 0.05 to 0.25 . We use an invasion percolation based model which has been shown to properly reproduce displacement patterns observed in previous experiments. We present 
a quantitative analysis of the size distribution of trapped fluid clusters. We show that when the in-plane curvature is considered, the amount of trapped fluid mass first increases with increasing correlation scale $L_{c}$ and then decreases as $L_{c}$ further increases from some intermediate scale towards the domain length scale $L$. The in-plane curvature contributes to smoothening the invasion front and to dampening the entrapment of fluid clusters of a certain size range that depends on the combination of random aperture standard deviation and spatial correlation.

Keywords: fracture, two-phase flow, drainage, curvature, invasion percolation, fluid trapping

\section{Introduction}

Many important subsurface engineering applications, such as the geological storage of $\mathrm{CO}_{2}$, nuclear waste disposal, and geothermal exploitation, involve fractured media and give rise to two-phase flow phenomena in fractures. While understanding the two-phase flow behavior at the scale of fracture networks is important for practical applications, modeling at such a scale requires fundamental knowledge of the behavior at the singlefracture scale. Of particular importance is the fluid trapping process during immiscible displacement in the open fracture. The present study focuses on characterizing fluid trapping and examining its controlling parameters.

The general problem of fluid trapping in geological media is central for engineering situations such as petroleum recovery and $\mathrm{CO}_{2}$ sequestration. In the former situation, the trapping of oil or gas in the pore space should be minimized to achieve the recovery efficiency of the reservoir. In the latter, on the contrary, trapping of supercritical $\mathrm{CO}_{2}$ in the pore space by various mechanisms is desired as it improves storage capacity and 
safety. Recently there has been extensive investigation of fluid displacement and trapping in porous material, including both pore-scale numerical modeling $[7,18,23,36]$ and experimental studies (e.g., $[3,4,12,22,28,40])$ thanks to the advances in high-resolution imaging techniques (e.g., X-ray computed tomography). But, fluid trapping in fractures has received relatively little attention in the recent literature, although many of the above modeling and experimental methodologies can be applied to the fractured setting.

Natural rock fractures have rough surfaces and variable apertures. The aperture distribution is one of the primary parameters that influence the hydraulic properties of a fracture, both for single and multiphase flow processes. Considerable effort has been devoted to the characterization and measurement of fracture wall topographies and of the resulting fracture aperture fields. Different approaches for aperture measurement have been developed, including surface profilometry (e.g., $[1,11,38])$, X-ray computed tomography (e.g., [27]) and nuclear magnetic resonance imaging (e.g., [15]). The high resolution measurement of aperture fields together with modern visualization techniques has allowed for fundamental investigations of fluid displacement in single fractures. Those measurements and the subsequent analyses of surface topographies of natural fractures have established that these topographies are self-affine (e.g., $[8,10]$. Besides, the two walls of a fracture have topographies that are essentially identical at length scales larger than a typical mismatch (or correlation) length $L_{c}$, and uncorrelated with each other at scales smaller than $L_{c}[10]$. Consequently, the resulting aperture field is self-affine at scales smaller than $L_{c}$, and exhibit hardly no fluctuations at scales larger than $L_{c}[10,34]$.

Such a representation of fracture apertures, based on geostatistical parameters (e.g., mean aperture, standard deviation, and correlation length) quantifying the aperture 
distribution, has been used in several studies involving fluid flow in fractures $[5,9,32$ -

34,47]. It has also proven useful to understand the effect of variable apertures on singlephase flow and transport [26,29].

Configurations of immiscible two-phase flow in individual variable aperture fractures have also been addressed $[6,16,21]$. Such flows are controlled by the interplay between capillary, gravitational, viscous and inertia forces [14]. Understanding that interplay and its impact on the flow regimes and the resulting fluid phase configurations is a prerequisite to studying fluid trapping. Loggia et al. [30] showed that under the influence of buoyancy two-phase flow regimes range from tortuous fingers and random clusters to piston-like displacement with trapping, depending on different combinations of the dimensionless Bond numbers and capillary numbers, which compare buoyancy to capillary forces and viscous to capillary forces, respectively. However, the fracture geometries, flow conditions and regimes explored in these experiments are still limited in comparison to the wide spectrum of behaviors that can occur. On the other hand, insights from investigating fluid trapping as a function of the medium geometry and flow conditions for a given system could allow for an a posteriori characterization of the flow regimes, from the mere knowledge of the trapped cluster geometry. Trapping of the defending fluid during immiscible displacement affects the flow structure of the invading phase behind the front. The amount of trapped phase fluid and its spatial distribution within the fracture affects the relative permeability of the other fluid phase and the fluidfluid interfacial area. In order to estimate the interfacial area, the characteristics of the trapped phase, i.e., the morphology and topology of the trapped phase clusters, need to be 
known [19]. Interfacial area is an important parameter for the interphase mass transfer processes that are critical, e.g., to contaminant remediation problems $[13,43,45]$.

Quasi-static displacement in horizontal fractures is dominated by capillary forces, so that in models viscous forces and gravity can be ignored. The capillary number, which compares viscous to capillary forces, has to be much smaller than 1 in this case. Invasion percolation (IP) models have been widely recognized as a physically-sound approach for modeling such slow displacements in rough-walled fractures (see experimental and theoretical studies, e.g., $[2,17,20,24,31,35,44])$. In these models, a proper calculation of the capillary pressure between the wetting and non-wetting fluids is crucial when simulating fluid invasion, especially if one focuses on characterizing phase distribution and structures. According to the Young-Laplace equation, capillary pressure depends on the interfacial tension and the mean curvature of the fluid-fluid interface. For the fracture geometry, the mean curvature can be calculated using its two principal components, one of them being defined in a vertical plane, the other, which we shall denote in-plane curvature, being defined in the fracture plane. Displacement in the capillary regime is controlled by the competition between the effects of (i) the aperture variability along the fracture plane, which tends to roughen the interface, and (ii) the in-plane curvature, which tends to smoothen it [21]. To calculate the local in-plane curvatures, Glass et al. [20] proposed to use an empirical length scale (which the authors set to half of the correlation length, defined in terms of the autocorrelation or variogram of the aperture field) and an average angle extracted from the local fluid-fluid interface. Similar approaches have been used by Neuweiler et al. [35] and Ferer et al. [17] who treated the empirical length scale as a fitting parameter to be determined by trial and error based on a comparison with 
physical experiments, which is difficult to be generalized. To overcome those limitations of the above mentioned approaches, Yang et al. [42] developed a generalized method based on a purely geometric definition of the curvature radius, in consistency with the Laplace equation. They estimated the local in-plane curvature radius through a procedure that adaptively fits a circle to the fluid-fluid interface. The in-plane curvature is estimated as the inverse of the radius of the best fitted circle. This approach was validated against experimental data and shown to be advantageous over previous approaches (see 42). Another recent study [46] investigated the effect of aperture field geometry on relative permeabilities; however, the IP model used by Ye et al. [46] took neither in-plane curvature, nor the trapping of the defending fluid, into account.

To our knowledge, the effect of the aperture field geometrical (geostatistical) parameters on fluid displacement and trapping is still not fully understood. The purpose of this study is thus to quantitatively characterize the trapping of the defending wetting fluid during drainage, under various aperture field geometries. We focus on trapped wetting phase saturation and on the distributions of sizes for trapped fluid clusters. The aperture fields investigated span a range of geometries, from uncorrelated fields to fully self-affine fields.

\section{Method}

In this section, we will first present the method to generate realistic aperture fields, followed by the description of the model which is based on invasion percolation including the effect of in-plane curvature. Then, we describe the simulation scenario of nonwetting phase invasion and the parametric study design. 


\subsection{Fracture aperture fields}

To describe the topography fracture wall surfaces, we follow Brown [10] to define a power spectrum for surface roughness of the form

$$
G\left(\xi_{x}, \xi_{y}\right) \propto\left[\xi_{x}^{2}+\left(\xi_{y} / a\right)^{2}\right]^{-(1 / 2+H)}
$$

where $\xi_{x}$ and $\xi_{y}$ are the wave numbers in the $x$ and $y$ dimensions, $H$ is the Hurst exponent, in the range of $0.5<H<1$ with a typical value of $H=0.8$ [8], and $a$ the anisotropy factor. In this study, we assume isotropy in fracture surface roughness by assigning $a=1$. We use an inverse fast Fourier transform (iFFT) method to generate fracture wall surfaces.

Fracture apertures correspond to the gap between the two facing rough surfaces, whose average planes are parallel to each other. Experimental work by Brown et al. [11] has shown that at large wave numbers (short wave lengths) the power spectrum of fracture aperture fields has the power law behavior typical of self-affinity and described in Eq. (1), and therefore characterized by the corresponding Hurst exponent, but that at small wave numbers (long wave lengths) the spectrum density approaches a constant value. We define a mismatch (or cutoff) wave number (spatial frequency), $\xi_{c}$ (respectively, a mismatch length scale, $L_{c}$ ), below (respectively, above) which the spectrum density flattens out. To ensure the two surfaces to be matched at small wave numbers (i.e., long wave lengths), we generate the two surfaces with phase spectra that are identical below the cutoff wave number $\xi_{c}$. The resulting random aperture fields are self-affine at scales smaller than $L_{c}$, and exhibit hardly any heterogeneities at scales larger than $L_{c}$. A more detailed description of the procedure for generating the fracture surfaces and aperture fields can be found in Brown [10]. 


\subsection{Invasion percolation model with in-plane curvature}

We model the capillary displacement of a wetting fluid by a non-wetting fluid (drainage) inside a geological rough fracture. This means that we consider slow displacement in which viscous forces are too small to have any role in defining the geometry of the fluid-fluid interface as it travels along the fracture plane. Practically this implies that the capillary number $\mu_{i n v}\langle v\rangle / \gamma$ is sufficiently small, in any case much smaller than 1 . We also consider a fracture whose mean plane is horizontal, as a first step into the study of drainage in networks of fractures with various orientations.

Even in a fracture of horizontal mean plane, buoyancy forces could still play a role since the fracture walls are rough and their vertical position varies along the topography. In this case, whether buoyancy forces would impact the geometry of the interface or not can be assessed by comparing the fluctuations of hydrostatic pressure due to topographic fluctuations with fluctuations of capillary pressure that result from aperture variations. The ratio of those forces defines a non-standard dimensionless Bond number $N^{*}{ }_{B o}$, as follows:

$$
N *_{B o}=\frac{\Delta \rho g \Delta h}{\langle P c\rangle \delta}=\frac{\Delta \rho g\langle b\rangle \Delta h}{2 \delta \gamma \cos \theta}
$$

where $\Delta \rho$ is the fluid density difference, $\Delta h$ is the maximum elevation difference of the fracture surface fluctuation, $\delta$ is the coefficient of variation of the aperture field, $\gamma$ is the interfacial tension, $\theta$ is the contact angle and $\langle P c\rangle$ is the capillary pressure corresponding

to the mean aperture $\langle b\rangle,\langle P c\rangle \delta$ being the typical fluctuation of $P c$ around its mean value. For geological fractures, $\Delta h$ is typically on the order of a few millimeters (see, e.g., Brown et al., 1986). For example, if $\Delta h=5 \mathrm{~mm}, \Delta \rho=200 \mathrm{~kg} / \mathrm{m}^{3}, \delta=0.25$ and $\gamma=35$ 
$\mathrm{mN} / \mathrm{m}$, the Bond number $N^{*}{ }_{B o}$ is found to be around $N^{*}{ }_{B o} \approx 0.05 \ll 1$, which indicates that the impact of buoyancy forces on the interface geometry can be neglected for this parameter set. Note also that another buoyancy-driven process could occur: that the lighter displacing fluid (e.g., air) flow on top of the denser displaced fluid (e.g., water). It would be the case if the typical buoyancy pressure drop over the mean fracture aperture were much larger than $\langle P c\rangle$; the ratio of those forces yields the standard Bond number, defined at the scale of $\langle b\rangle: N_{B o}=\Delta \rho g\langle b\rangle /\langle P c\rangle=N{ }_{B o} \delta\langle b\rangle / \Delta h$, which is much smaller than $N^{*}{ }_{B o}$. Thus, the condition $N^{*}{ }_{B o} \ll 1$ is the only one required to ensure that buoyancy forces do not impact the interface geometry. This condition is obviously fulfilled for a variety of subsurface configurations, as shown above. However, if the fluid density difference is much higher or the aperture coefficient of variation much smaller, so that $N^{*}$ Bo becomes closer to 1 , buoyancy forces can have a significant impact. In this regard, we recognize the need for a full parametric study concerning the buoyancy effect in the future.

Our motivation is thus to understand the effects of the interplay of capillary forces and aperture structure on fluid phase configurations during two-phase flow along a fracture geometry such as presented in Section 2.1. In order to do this we use an Invasion Percolation (IP) model: the single fracture void space is conceptualized as a 2D domain representing the fracture plane, which is discretized into a 2D lattice with aperture values on all sites, assigned from the generated aperture spatial distribution. The discretization scale of the model is the size of the individual site, which in a $2 \mathrm{D}$ porous medium would typically be a pore of the medium. Therefore no corner flow or film flow can be described with an IP model. Nevertherless they have proved very useful at predicting the 
interface geometry in 2D porous media $[17,20,24,35,42]$. The fluid displacement process is modeled as a sequence of discrete invasion steps. Each of the invasion steps are determined by ranking of invasion pressures at all sites of the (invading-defending) phase interface; the site with the smallest invasion pressure is selected at each step. The invasion pressure $P_{i n v}$ for a given site is calculated according to the Young-Laplace equation as [42]:

$$
P_{i n v}=2 \gamma H=\gamma\left(k_{1}+k_{2}\right)=\gamma\left(\frac{1}{r_{1}}+\frac{1}{r_{2}}\right)=\gamma\left(\frac{2 \cos \theta}{b}+\frac{1}{r_{2}}\right)
$$

where $\gamma$ is the interfacial tension, $H$ is the mean curvature, $k_{1}$ and $k_{2}$ are the two principal curvatures, calculated respectively as the inverse of the two principal radii of curvature $r_{1}$ and $r_{2}\left(k_{l}=1 / r_{1}\right.$ and $\left.k_{2}=1 / r_{2}\right)$. Fig. 1 shows a schematic of the interface curvatures. The plane for the principal curvature $k_{2}, \mathrm{P} 2$, is the plane of the fracture, while the plane for $k_{l}$, $\mathrm{P} 1$, is perpendicular to $\mathrm{P} 2$. The cut of the fluid-fluid interface by $\mathrm{P} 1$ produces a curve (shown in Figure 1(a)) that spans the fracture aperture, while the cut of the interface by P2 produces a curve (shown in Figure 1(b)) that is within the fracture plane. Following earlier works $[20,35,42]$, we refer to $k_{l}$ as out-of-plane (aperture spanning) curvature and to $k_{2}$ as in-plane curvature.

If we assume that the fracture walls are symmetric about a mean plane, $r_{l}$ can be calculated as $b / 2 \cos \theta$, where $b$ is the local aperture, and $\theta$ the contact angle. Note that here we ignore the local convergence/divergence angle of the fracture walls [35]. The out-of-plane curvature $1 / r_{l}$ can be pre-assigned for all sites before starting the IP invasion process. However, the in-plane curvature is dependent on the interface configuration along the fracture plane (P2) and needs to be calculated at each invasion step for all sites belonging to the interface at that time. In this the modeling of capillary displacement in a 
fracture is drastically different from the standard IP modeling of capillary displacement in 2D porous media, which have been the subject of a vast amount of literature; indeed, in standard IP the displacement is completely controlled by the quenched-disorder in the pore throat sizes, as the invasion pressure is reduced to a single term analog to the out-ofplane curvature term in Eq. (3).

To calculate the in-plane curvature, we use the approach of Yang et al. [42] which is based on purely geometric arguments and implements a procedure called adaptive circle fitting to determine the varying in-plane curvature during fluid displacement. The inplane curvature is calculated as the inverse of the radius of the best fit circle to the local interface. This is done by nonlinearly fitting the coordinates of the appropriately chosen interface sites to the circle equation [42]:

$$
f(x, y)=\left(x-x_{c}\right)^{2}+\left(y-y_{c}\right)^{2}-z^{2}=0
$$

where $x_{c}, y_{c}$ and $z$ are fitting parameters, $x_{c}$ and $y_{c}$ denoting the coordinates of the center of the fitted circle, and $z$ denoting the radius of the fitted circle. We adaptively determine the number of interface sites to be used for fitting the circle equation, by keeping track of the normalized fitting residuals. A full description of the approach can be found in Yang et al. [42].

The trapping of the defending-phase is implemented assuming that the defending phase is incompressible; that is, the defending phase occupied sites that become surrounded by the invading phase, i.e., the sites that have lost connection to the outlet boundaries through the defending phase, can no longer be invaded. This is implemented through an efficient bidirectional search algorithm to determine whether a site or region is connected to the outlet boundary. 


\subsection{Simulation scenario and parameters}

We consider horizontal fractures, and assume that the effect of gravity can be neglected. We simulate drainage, that is, we model the capillary dominated displacement of a wetting phase by a nonwetting phase. We use a 2D rectangular domain of $2048 \times$ 1024 sites with the left short edge assigned to be the nonwetting source boundary and the other three edges assigned to be open boundaries where the wetting phase is allowed to leave the domain (Fig. 2). The wetting phase fluid may be trapped if surrounded by the invading nonwetting phase fluid. We consider a perfect wetting condition for the fracture surfaces. The contact angle is set to zero; for a contact angle that is uniform over the medium, this can be done without loss of generality). The interfacial tension is chosen equal to $34.5 \mathrm{mN} / \mathrm{m}$. We note that increasing the contact angle will have the same effect on the displacement as decreasing aperture field standard deviation, , since $r_{l}=b / 2 \cos \theta$ and $r_{2}$ is independent of $\theta$. The spatial discretization is $d=0.1 \mathrm{~mm}$. The Gaussian random aperture fields are generated with the method described in Section 2.1. The Hurst exponent is set to $H=0.8$ and the mean aperture $\langle b\rangle$ to $0.1 \mathrm{~mm}$. We vary the coefficient of variation of the aperture field, $\delta=\sigma_{b} /\langle b\rangle$, where $\sigma_{b}$ is the aperture standard deviation, from 0.05 to 0.25 . We also vary the mismatch wave number $\xi_{c}$, from 1 to 256 , with $\xi_{c}=1$ representing a limiting case where the aperture field is self-affine on the entire available scale range. We define the aperture correlation scale as $L_{c}=L / \xi_{c}, L$ being the domain length $2048 d$. Fig. 3 shows six example realizations of generated aperture fields. One could argue that the horizontal discretization length, $d$, introduces an additional length scale in the system. One could consider it as a lower limit cutoff scale for pattern sizes. However, as $d$ is equal to the mean aperture, $\langle b\rangle$, patterns on sizes smaller than that size 
are expected to be smoothed out by in-plane curvature, as long as the coefficient of variation is not very large, i.e., $\delta \leq 0.25$. For each parameter set, ten realizations are generated and resulting fluid configurations are simulated. We add a case with spatially uncorrelated random aperture fields which can be thought of as $\xi_{c}=2048$. Another limiting case in which the in-plane curvature is not taken into account in the calculation of the capillary pressures (in effect this model is essentially the standard IP/model with trapping) is also considered. The model is run until all sites are either invaded or trapped. The total number of simulations is 480 .

\section{Results and analysis}

Residual saturation is an important macroscopic property for describing two-phase flow in porous and fractured media. Our simulations exhibit trapping of the wetting phase, which results in an irreducible wetting phase saturation in the fracture. In this section, we first present qualitative examination of the trapped phase morphology, then we proceed to quantify the macroscopic trapped phase saturation and analyze the size distributions of the trapped fluid clusters in detail.

\subsection{Trapped phase patterns}

Fig. 4 shows six example spatial distributions of the trapped wetting phase within the fracture for three different aperture correlation scales $L_{c}$ and two different aperture coefficients of variation $\delta$. (More trapped phase patterns can be found in the Supporting information, Fig. S1 and Fig. S2.) Through the comparison between Fig. 4(a-c) and Fig. 4(d-f), it can be seen that both the number of trapped clusters and the total trapped mass tend to be smaller as $\delta$ is smaller. It can also be observed that the difference in trapped 
phase distribution between the two $\delta$ cases becomes more drastic as $L / L_{c}$ becomes larger (correlation scale smaller). Simulation results with parameters $\delta=0.25$ and $L / L_{c}=256$ (see Fig. 4c) show a significant mass of trapped wetting fluid (corresponding to a trapped saturation $S_{t}=0.42$ ) as well as a wide range of trapped cluster sizes, spanning five orders of magnitudes from a few site sizes to $\sim 10^{5}$ site sizes. When $\delta=0.00833$ and $L / L_{c}=256$ (Fig. 4f), the amount of trapped mass is almost negligible $\left(S_{t}=0.01\right)$. It should be noted that this is an effect of the in-plane curvature. An IP algorithm without in-plane curvature would yield exactly the same invasion patterns for two geometries generated with the same correlation length and numerical seed, but different coefficients of variation.

The above qualitative observations suggest that variability and spatial correlation of fracture apertures play a critical role in the fluid-fluid displacement and fluid trapping process. Greater variability (larger $\delta$ ) consistently produces a larger total mass trapped fluid for all investigated ratios of the system length to the correlation length. This is due to the effect of the in-plane curvature which smoothens the invading front. Indeed, trapping occurs when two portions of the interface positioned ahead of the rest of the interface meet and merge, leaving a closed interface loop behind; hence, a rougher interface results in more trapping of the defending phase. The smoothing effect of the inplane curyature being weaker when the aperture variability is larger [21], larger values of $\delta$ exhibit a rougher interface and therefore a larger total mass of trapped defending fluid. However, the influence of the correlation length on fluid trapping remains unclear in Fig. 4 and needs a more detailed examination (which is addressed in the following sections). 


\subsection{Trapped phase saturation}

Fig. 5 presents the trapped (irreducible) saturation averaged over results from realizations of statistically-equivalent aperture fields. The curves of trapped phase saturation plotted against $\langle b\rangle / L_{c}$ for different $\delta$ may be regarded as type curves when an estimation of trapped phase saturation, given aperture variability and correlation scale, is needed. Fig. 5 shows that the trapped phase saturation is at its maximum when the effect of in-plane curvature is not taken into account in the model (the IP limit). Also evident is the increasing trend of trapped phase saturation with decreasing aperture correlation scale (increasing $\langle b\rangle / L_{c}$ ) in the IP limit (blue circle plot), from $\left\langle S_{t}\right\rangle=0.32$ for the self-affine case $\left(\langle b\rangle / L_{c}=1 / 2048\right)$ to $\left\langle S_{t}\right\rangle=0.69$ for the case with no spatial correlation $\left(L_{c}=\langle b\rangle=\right.$ d). This increasing trend of the irreducible saturation as a function of $\langle b\rangle / L_{c}$ for standard IP agrees with Knackstedt et al. [25] who simulated invasion with an IP model on correlated 3D lattice. Naturally, the trivial lower bound for trapped phase saturation is zero, which can be achieved when $\delta \rightarrow 0$ (ideal parallel-plate fracture).

It is clear from Fig. 5 that the trapped saturation decreases with decreasing $\delta$. On the other hand, the dependence of the trapped phase saturation on $\langle b\rangle / L_{c}$ for a given $\delta$ is not as straightforward. The maximum of $\left\langle S_{t}\right\rangle$ for a given $\delta$ is at some intermediate value of $\langle b\rangle / L_{c}$. It can be said that the value of $\langle b\rangle / L_{c}$ at which $\left\langle S_{t}\right\rangle$ is maximum for a given $\delta$ is smaller when $\delta$ is smaller. More discussion will follow on this in Section 4.

To investigate the general behavior described above in more detail, we study the size distribution of the trapped fluid clusters and the contribution of each class of cluster sizes to the trapped phase saturation. 


\subsection{Trapped fluid cluster size distributions}

For each simulation, we consider the final configuration of fluid phase distribution and extract all the clusters of trapped fluid from it. We then compute a histogram of the cluster sizes, that is, we divide the overall cluster size range in 40 classes of identical width and count the number of clusters $n(s)$ for each class (centered on cluster size $s$ ). By size we mean the number of lattice sites occupied by the cluster. We finally put together the cluster size statistics from all the realizations corresponding to a given aperture field parameter set, in order to investigate the influence of aperture variation and correlation scale on trapped cluster size distribution.

\subsubsection{IP model not taking in-plane curvature into account}

For a square lattice, percolation theory [37,39] has established that at the critical point, the cluster size distribution for a percolation system without trapping effect follows a power law, $n(\mathrm{~s}) \sim s^{-\tau}$, with a theoretical value of 187/91 for the Fisher exponent $\tau$. However, when trapping is considered in the system, the cluster size distribution behavior will be different. The pioneering work of Wilkinson and Willemsen [41] has estimated the power law exponent for trapped cluster size distribution resulting from IP with trapping effect for different lattices using simulations.

Fig. 6a shows that in the case of an uncorrelated random aperture field the trapped cluster size distribution follows a power law behavior. We perform linear fitting (See Supporting Information Fig. S3) to the cluster size data of the uncorrelated cases and find the exponent $\tau$ of this power law to be $1.85 \pm 0.02$ (95\% confidence interval), which is in agreement with the result (1.84) obtained by Wilkinson and Willemsen [41]. When there is spatial correlation in the aperture field, the cluster size distribution follows the same 
power law $n(\mathrm{~s}) \sim s^{-1.85}$ at sizes larger than a crossover size $s_{c}$ (which scales with the correlation length as $s_{c} \sim L_{c}{ }^{2}$ ), but deviates negatively from it at sizes smaller than $s_{c}$, which means that we obtain less clusters of sizes smaller than $s_{c}$. At much smaller cluster sizes the cluster size distribution displays a power law again, which results from the trapping of defendinging fluid due to aperture variations over horizontal lengths smaller than the correlation scale (sub-correlation scale trapping). Note that for geometries corresponding to $L / L_{c}<64$, this shifting of the cluster size distribution around $s_{c}\left(\sim L_{c}^{2}\right)$ happens in the tails and is thus not visible in Fig. 6a. But plotting the cumulative size distribution $N\left(s^{\prime}>s\right)$ against $s$ evidences this transition for all $L / L_{c}$ values (Fig. 7, left). In Fig. 7(right) we also show the collapse of the cumulative cluster size distribution curves of Fig. 7(left); the shifting behavior consistently happens at the same scale $\pi^{0.5} L_{c}$. A single scaling relates $N\left(s^{\prime}>s\right)$ to $s$ according to $N\left(s^{\prime}>_{S}\right)=\left(L / L_{c}\right)^{\alpha} \cdot \mathrm{f}\left((s / \pi)^{0.5} / L_{c}\right)$, the exponent $\alpha$ is found empirically to be 1.70 , and $f$ is a master curve function represented in Fig. 7(right).

Because of the above-mentioned transition at $s_{c}$, we expect the total amount of trapped mass

$$
M=\sum_{s \geq 1} \operatorname{sn}(s)
$$

to be smaller when $L_{c}$ is larger. This explains the increasing trend for the IP limit (blue circle plot) in Fig. 5.

\subsubsection{IP including in-plane curvature}

Inclusion of the effect of in-plane curvature in the model significantly changes the cluster size distribution (Fig. 6b-f). When $\delta \geq 0.125$, it seems that for uncorrelated fields IP considering in-plane curvature produces trapped cluster size distributions that follow a 
power law, but with different exponents $(<1.85)$ than in the case without in-plane curvature. When $\delta<0.125$, IP with in-plane curvature for uncorrelated fields produces no or very few small trapped clusters, meaning that the irreducible saturation is essentially zero.

For the correlated fields with a high coefficient of variation $(\delta \geq 0.125)$, the cluster size distribution starts above the curve corresponding to uncorrelated aperture field at some cluster size and then goes below the curve corresponding to uncorrelated aperture field at a smaller cluster size. This means that there are more large clusters but less small clusters than for the uncorrelated case. In contrast to the cases of IP not considering inplane curvature, sub-correlation scale trapping is dampened due to the smoothing effect of the in-plane curvature, and the dampening effect is stronger when $L_{c}$ is larger.

For the correlated fields with a low coefficient of variation $(\delta \leq 0.0833)$, as shown in Fig. 6d-f, the smoothing effect of the in-plane curvature is relatively strong and results in significantly less trapped clusters. This dampening effect is observed both at small cluster sizes and at large cluster sizes, which results from the suppressed trapping of small clusters. The dampening increases with decreasing coefficient of variation, until basically no trapping occurs any more.

It is of interest to apply the same scaling relation as used in Fig. 7 to the cases where in-plane curvature is considered. Fig. 8 presents the cumulative cluster size distribution for $\delta=0.25$ before and after using the scaling relation with $L_{c}$. There is obviously a cutoff to the power law behavior around the correlation scale. For cases with smaller $\delta$, the simple scaling relation does not collapse the curves (figures not shown). This is due to the 
much stronger dampening effect of the in-plane curvature for both clusters below and beyond the correlation scale. This cutoff is discussed in more detail in Section 4 .

\subsection{Cluster size composition of the trapped phase fluid}

In order to look at the contribution of trapped clusters of different sizes to the total trapped mass, we plot normalized trapped saturation from clusters with sizes larger than or equal to a certain size $s$ (Fig. 9). This conditional normalized trapped saturation is calculated as $\sum s^{\prime} n\left(s^{\prime} \geq s\right) / \sum s n(s)$. It can be observed that when the in-plane curvature is considered, the curves for the long-correlated (self-affine) field and the short- or uncorrelated field create an envelope. When the correlation scale is larger, the irreducible saturation is more dominated by the few large clusters. This is expected as spatial correlations exist in the aperture field up to the fracture scale, and allow for the existence of correlated regions of close-to-uniform aperture and of lateral extension of the same order as the system size. In those regions the trapping of wetting fluid clusters of size similar to the region size is possible.

We can also define a cutoff cluster size such that the majority (say, 90\%) of the irreducible saturation is distríbuted in trapped clusters with sizes larger than this cutoff size. Fig. 10 presents the $90 \%$ cutoff cluster size for all cases. Generally, when the inplane curvature is considered, the cutoff size increases with increasing correlation scale. This is because longer correlation length leads to more dampening of trapping of small clusters under the influence of in-plane curvature (see Fig. 6b-f). But when the in-plane curvature is neglected (i.e., in the IP limit), the cutoff size is largest at an intermediate correlation scale $\left(L / L_{c}=64\right)$. This can be explained by (i) the decreasing trend of the total trapped mass with increasing correlation length and (ii) the contribution of the sub- 
structure trapping below the correlation scale to the total trapped mass in the fracture.

When $L / L_{c}$ decreases from 2048 to 64 , the total trapped mass decreases because there are fewer trapped clusters of sizes below the correlation scale. But when $L / L_{c}$ further decreases, the contribution of sub-correlation scale trapped mass becomes larger than $10 \%$, causing the $90 \%$ cutoff size to be smaller than that for $L / L_{c}=64$. If the threshold value would have been chosen differently from $90 \%$, the correlation length scale having the largest cutoff cluster size would be different.

\section{Discussion}

We have investigated the effect of the aperture field's geometry on capillary dominated displacement and trapping in fractures. The problem setup is similar to that of Glass et al. [21]. The simulation results show that the amount of trapped fluid depends on whether the in-plane curvature, that is, the minor principle component of the interface curvature, parallel to the fracture plane, is táken into account in the numerical model. The in-plane curvature acts to smooth the interface and decrease trapping. Generally, the smaller the coefficient of variation, the less the defending phase fluid can be trapped. This part of the results is in agreement with Glass et al. [21].

However, when it comes to the effect of aperture correlation scale, we have observed a different trend in trapped phase saturation than the one suggested in Glass et al. [21]. It is implied in Fig. 4 of Glass et al. [21] that trapped phase saturation increases monotonically with increasing correlation length. However, our results suggest that, when the in-plane curvature is considered in the model, the amount of trapped fluid mass first increases with increasing correlation scale $L_{c}$ and then decreases as $L_{c}$ further increases from some intermediate scale towards the domain size (Fig. 5). That is to say, when the in-plane 
curvature is considered and the coefficient of variation is fixed, the trapped phase saturation is highest at some intermediate correlation scale, given a finite domain size.

The discrepancy between our results and those of Glass et al. [21] stems from the different approaches used for calculating the in-plane curvature. Our model calculates the in-plane curvature as a purely geometrical parameter, by local fitting of osculating circles to the interface; in this, it closely follows the definition of curvature. The model used in Glass et al. [20,21] involves pre-defining a length scale (half of the correlation length) for the calculation of the in-plane curvature. In fact, according to Glass et al. [21], when the correlation length is much larger than the mean aperture $\left.\left(L_{c}\right\rangle\langle b\rangle\right)$, the invasion conforms to the IP limit. However, the IP limit would imply an invading front that is nonsmooth at any length scale, which then would be inconsistent with their model assumption that the in-plane radius is of the order of half the correlation length.

The dependence of the global trapped saturation $S_{t}$ on the coefficient of variation $\delta$ of the apertures can in general be explained. The trapping effect is a competition between the pinning of the interface at a location of small apertures and the pinning induced inplane curvature. By pinning we mean here that the interface/front tends to be slowed down or even arrested for a while at locations of small aperture, by the part of the capillary force that stems from the out-of-plane curvature. This impeding action by the small aperture leads to roughening of the interface, while the in-plane curvature counteracts to smoothen. One could imagine a threshold aperture, smaller than the mean aperture $\langle b\rangle$, above which the local front is smoothed out by the in-plane curvature and below which it is not. It is clear that an aperture field with a smaller coefficient of variation has less such apertures than a field with higher coefficient of variation (See Fig. 
11). This explains that there is in general less trapping in fields with smaller coefficients of variation (Fig. 5).

The dependence of $S_{t}$ on the aperture correlation scale is more subtle. Fig. 5 shows that $S_{t}$ increases with decreasing $L_{c}$ for large correlation lengths (note that we plot $S_{t}$ against $\langle b\rangle / L_{c}$; when $L=L_{c}$, as in the case of self-affine aperture field, $\langle b\rangle / L_{c}$ is at its minimum 1/2048). This behavior can be explained with the following reasoning. For a given $\delta$, the area occupied by small apertures (below a threshold aperture) is the same for different correlation scales, and one may regard the small-aperture regions as a source leading to trapping. For large correlation lengths (low $\langle b\rangle / L_{c}$, left side of Fig. 5), the dominant features of the fluid-fluid interface roughness have a length scale corresponding to the correlation length. As the correlation length decreases from $L_{c}=L$, two observations can be made (compare Fig. 11b and Fig. 11c): (i) the smaller the correlation length, the larger the number of clusters of small apertures that form loops with large-aperture clusters inside; these large-aperture clusters will be consequently inaccessible to the invading front and (ii) more channels of large apertures are available for a given spatial scale, meaning that more invasion pathways are possible. These two concomitant effects of decreasing $L_{c}$ lead to increased probability of by-pass trapping with trapped clusters of size beyond the correlation scale (which means that the probability of trapping largeaperture sites is higher). This explains that the total trapping increases with decreasing $L_{c}$, for large $L_{c}$ values.

However, as the correlation length is decreased further and approaches the uncorrelated case (right side of Fig. 5), the size range of the loops (of small apertures) widens and the small loops below a certain size will be smoothed out by the in-plane 
curvature. Thus, at some intermediate $L_{c}$ value (depending also on $\delta$ ), the total area of trapped clusters starts to decrease with further decrease in correlation length.

For a field with a low variance only few apertures with a value below a threshold exist. When the correlation length also becomes small, these apertures only generate isolated small spots (see Fig. 11d). However, these small spots, giving rise to small inplane radii at the interface, will not lead to trapping of the defending fluid, because the interface roughness at these spots will be smoothed out by the in-plane curvature. For this reason, the trapping is strongly suppressed in fields with both a small $\delta$ and a small $L_{c}$ (Fig. $4 f$ and Fig. 5).

We can also make a note about the aperture variability (coefficient of variation). As the correlation scale increases towards the domain length scale, the large aperture regions and small aperture regions are more and more segregated into large patches. As a result, when the defending fluid is displaced, the apertures 'sampled' by the invading front will have a significantly narrower distribution (smaller coefficient of variation locally) than the global aperture field. This also supports to explain why the trapped phase saturation drops when the aperture correlation shifts from an intermediate scale towards the selfaffine fields. Therefore, it may be useful to define an effective coefficient of variation $\delta_{f}$ (as opposed to the global apparent coefficient of variation $\delta$ ) calculated using the aperture values of the sites at the invading front. This motivates further studies.

\section{Conclusions}

Geostatistical characteristics of the aperture field in a rough-walled fracture have a strong impact on the two-phase fluid displacement and the resulting fluid entrapment. Our work has elucidated how the aperture correlation length and the coefficient of variation affect 
the fluid displacement and trapping processes in the capillary dominated regime. We have shown that when the in-plane curvature is not considered, and for the fully uncorrelated case, the trapped cluster distribution scales as a power law. When the inplane curvature is not considered and spatial correlation exists in the aperture field, the cluster size distribution follows the same power law as in the uncorrelated field case only for clusters of linear size larger than the correlation length $L_{c}$. When the in-plane curvature is taken into account and the aperture field is uncorrelated, the cluster size distribution also follows a power law, but with a different exponent. In addition, accounting for the in-plane curvature suppresses the formation of trapped clusters of size below the correlation scale. This dampening effect is strongly affected by the coefficient of variation $\delta$; the smaller the $\delta$, the smaller the number of trapped clusters and the total mass (or saturation) of trapped fluid. The dampening is also affected by the correlation length scale. Interestingly, when the in-plane curvature is considered, the trapped phase saturation is highest at some intermediate correlation scale, for a given aperture coefficient of variation.

The current work has also studied the composition of trapped mass in terms of the contribution from different cluster sizes. It has shown that, when the correlation scale is larger, the irreducible saturation is more dominated by a few large clusters, i.e., removal of the few large clusters will reduce most of the trapped mass. This has implications in situations where it is of interest to consider the subsequent interphase mass transfer process (e.g., evaporation of water, dissolution of supercritical $\mathrm{CO}_{2}$ or non-aqueous phase liquids) following fluid trapping. Generally smaller clusters have larger specific interfacial area, which means that the mass partitioning process is faster for smaller 
clusters. A typical scenario would be: the small clusters disappear at a relatively early stage and the large clusters undergo a slow partitioning process and create long-term tailing of smaller mass transfer fluxes.

In summary, we have presented a numerical investigation on the impact of aperture field geometry on capillary dominated displacement and fluid trapping. Despite the fact that only capillary forces are considered (no interplay between capillary, viscous and gravitational forces), a wide spectrum of entrapment morphologies can occur, spanning morphologies consisting of trapped clusters with a power law size distribution, to morphologies consisting of only a few sparse large clusters, and even to situations exhibiting no wetting fluid entrapment. The effect of in-plane curvature is to smoothen the invasion front and to dampen the entrapment of fluid clusters of a certain size range. This size range depends on the combination of the amplitude of the random aperture variability and aperture spatial correlation length. While the modeling results in this study are discussed in the context of rock fractures, the understanding of fluid displacement and trapping may as well apply to hydraulic properties of other two-dimensional thin-space flow devices. By choosing the aperture distribution (or alternatively the wettability of the confining walls) and the spatial correlation, one may be able to control how much fluid is to be trapped and according to what range of cluster sizes.

\section{Acknowledgments.}

Financial support for this work was provided by the Swedish Research Council (VR) International Postdoc Program, the EU FP7 project PANACEA, and the VR Strategic Funds for Energy Research. 


\section{References}

[1] Ameli P, Elkhoury JE, Detwiler RL. High-resolution fracture aperture mapping using optical profilometry. Water Resour. Res. 2013;49:7126-32. doi:10.1002/wrcr.20501.

[2] Amundsen H, Wagner G, Oxaal U, Meakin P, Feder F, Jøssang T. Slow two-phase flow in artificial fractures: Experiments and simulations. Water Resour. Res. 1999;35(9):2619-26. doi:10.1029/1999WR900147.

[3] Andrew MG, Bijeljic B, Blunt MJ. Pore-scale imaging of trapped supercritical carbon dioxide in sandstones and carbonates. Int. J. Greenhouse Gas Control 2014;22:1-14. doi:10.1016/j.ijggc.2013.12.018.

[4] Armstrong RT, Ott H, Georgiadis A, Rücker M, Schwing A, Berg A. Subsecond porescale displacement processes and relaxation dynamics in multiphase flow, Water Resour. Res. 2014;50: 9162-76. doi:10.1002/2014WR015858.

[5] Auradou H. Influence of wall roughness on the geometrical, mechanical and transport properties of single fractures. Journal of Physics D: Applied Physics, 2009;42(21):214015.

[6] Auradou H, Måløy KJ, Schmittbuhl J, Hansen A. Drainage in a rough gouge-filled fracture. Transport Porous Media, 2003;50:267-305. Doi:10.1023/A:1021164109061.

[7] Bandara UC, Tartakovsky AM, Palmer BJ. Pore-scale study of capillary trapping mechanism during $\mathrm{CO}_{2}$ injection in geological formations. Int J Greenhouse Gas Control 2001;5(6):1566-77. Doi:10.1016/j.ijggc.2011.08.014.

[8] Boffa JM, Allain C, JP Hulin. Experimental analysis of fracture rugosity in granular and compact rocks. Eur Phys J Appl Phys 1998;2:281-289. doi:10.1051/epjap:1998194. 
[9] Brown SR. Fluid flow through rock joints: the effect of surface roughness. Journal of Geophysical Research: Solid Earth 1987;92(B2):1337-47.

Doi:10.1029/JB092iB02p01337.

[10] Brown SR. Simple mathematical model of a rough fracture. J. Geophys. Res. 1995;100(B4): 5941-52. doi:10.1029/94JB03262.

[11] Brown SR, Kranz RL, Bonner BP. Correlation between the surfaces of natural rock joints. Geophys. Res. Lett. 1986;13:1430-33. Doi:10.1029/GL0131013p01430.

[12] Chaudhary K, Bayani Cardenas M, Wolfe WW, Maisano JA, Ketcham RA, Bennett PC, Pore-scale trapping of supercritical $\mathrm{CO} 2$ and the role of grain wettability and shape. Geophys. Res. Lett. 2013;40:3878-82. doi:10.1002/grl.50658.

[13] Detwiler RL, Rajaram H, Glass RJ. Nonaqueous-phase-liquid dissolution in variable-aperture fractures: Development of a depth-averaged computational model with comparison to a physical experiment. Water Resour. Res. 2001;37(12):3115-29. Doi:10.1029/2000WR000161.

[14] Detwiler RL, Rajaram H, Glass RJ. Interphase mass transfer in variable aperture fractures: Controlling parameters and proposed constitutive relationships. Water Resour. Res.2009;45(8):W08436. doi:10.1029/2008WR007009.

[15] Dijk P, Berkowitz B, Bendel P. Investigation of flow in water-saturated rock fractures using nuclear magnetic resonance imaging (NMRI). Water Resour. Res. 1999;35(2):347-60. doi:10.1029/1998WR900044.

[16] Dou Z, Zhou Z, Sleep BE. Influence of wettability on interfacial area during immiscible liquid invasion into a 3D self-affine rough fracture: lattice Boltzmann 
simulations. Advances in Water Resources. 2013;61:1-11.

doi:10.1016/j.advwatres.2013.08.007.

[17] Ferer M, Crandall D, Ahmadi G, Smith DH. Two-phase flow in a rough fracture: Experiment and modeling. Physical Review E, 2011;84(1):16316. doi:10.1103/PhysRevE.84.016316.

[18] Ferrari A, Lunati I. Direct numerical simulations of interface dynamics to link capillary pressure and total surface energy, Advances in Water Resources 2013;57:19-31. doi:10.1016/j.advwatres.2013.03.005.

[19] Geistlinger H, Mohammadian S, Schlueter S, Vogel H-J. Quantification of capillary trapping of gas clusters using X-ray microtomography. Water Resour. Res. 2014;50:4514-29. doi:10.1002/2013WR014657

[20] Glass RJ, Nicholl MJ, Yarrington L. A modified invasion percolation model for lowcapillary number immiscible displacements in horizontal rough-walled fractures: Influence of local in-plane curvature. Water Resour. Res. 1998;34(12):3215-34. (Correction, Water Resour.Res.2000;36(7):1991. doi:10.1029/2000WR900060.)

[21] Glass, RJ, Rajaram H, Detwiler RL. Immiscible displacements in rough-walled fractures: Competition between roughening by random aperture variations and smoothing by in-plane curvature. Physical Review E 2003;68: 061110. http://dx.doi.org/10.1103/PhysRevE.68.061110.

[22] Herring AL, Harper EJ, Andersson L, Sheppard A, Bay BK, Wildenschild D. Effect of fluid topology on residual nonwetting phase trapping: implications for geologic CO2 sequestration. Adv. Water Resour. 2013;62:47-58.

Doi:10.1016/j.advwatres.2013.09.015. 
[23] Jettestuen, E., Helland JO, Prodanovic M. A level set method for simulating capillary-controlled displacements at the pore scale with nonzero contact angles. Water Resour. Res. 2013;49:4645-61. doi:10.1002/wrcr.20334.

[24] Karpyn, ZT, Piri M. Prediction of fluid occupancy in fractures using network modeling and x-ray microtomography. I: Data conditioning and model description. Physical Review E 2007;76:16315. doi:10.1103/PhysRevE.76.016315,

[25] Knackstedt MA, Marrink SJ, Sheppard AP, Pinczewski WV, Sahimi M. Invasion Percolation on Correlated and Elongated Lattices: Implications for the Interpretation of Residual Saturations in Rock Cores. Transp. Porous Media 2001;44(3):465-85. doi:10.1023/a:1010770010309.

[26] Keller, AA, Roberts PV, Blunt MJ. Effect of fracture aperture variations on the dispersion of contaminants. Water Resour. Res. 1999;35(1):55-63. doi:10.1029/1998wr900041.

[27] Keller A. High resolution, non-destructive measurement and characterization of fracture apertures, International Journal of Rock Mechanics and Mining Sciences 1998;35(8):1037-50. doi:10.1016/s0148-9062(98)00164-8.

[28] Krevor S, Pini R, Zuo L, Benson S. Relative permeability and trapping of CO2 and water in sandstone rocks at reservoir conditions. Water Resources Research 2012;48:W02532. Doi:10.1029/2011WR010859.

[29] Lee J, Kang JM, Choe J. Experimental analysis on the effects of variable apertures on tracer transport. Water Resour. Res. 2003;39(1):1015. doi:10.1029/2001wr001246. 
[30] Loggia D, Bo Z, Luo X, Vasseur G. Experimental Study of Upward Oil Migration in a Fracture. Transport in Porous Media 2009;80(1):1-16. doi : 10.1007/s11242-0099340-2.

[31] Meakin P, Birovljev A, Frette V, Feder J, Jøssang T. Gradient stabilized and destabilized invasion percolation. Physica A 1992;191:227-39. Doi:10.1016/03784371(92)90532-U.

[32] Méheust Y, Schmittbuhl J. Flow enhancement of a rough fracture. Geophysical Research Letters 2000;27(18):2989-92. Doi:10.1029/1999GL008464.

[33] Méheust Y, Schmittbuhl J. Geometrical heterogeneities and permeability anisotropy of rough fractures. J. Geophys. Res. 2001;106 (B2):2089-102.

Doi:10.1029/2000JB900306.

[34] Méheust Y, Schmittbuhl J. Scale effects related to flow in rough fractures. Pure and Applied Geophysics 2003;160(5-6):1023-50. Doi:10.1007/PL00012559.

[35] Neuweiler I, Sorensen I, Kinzelbach W. Experimental and theoretical investigations of drainage in horizontal rough-walled fractures with different correlation structures. Adv. Water Resour. 2004;27(12):1217-31. doi:10.1016/j.advwatres.2004.07.005.

[36] Porter ML, Schaap MG, Wildenschild D. Lattice-Boltzmann simulations of the capillary pressure-saturation-interfacial area relationship for porous media. Adv Water Resour 2009;32(11):1632-40. Doi:10.1016/j.advwatres.2009.08.009.

[37] Sahimi M. Flow and transport in porous media and fractured rock. 718 pp., WileyVCH Verlag, Weinheim, Germany, 2011.

[38] Schmittbuhl J, Vilotte JP, Roux S. Reliability of self-affine measurements. Physical Review E 1995;51(1);131. http://dx.doi.org/10.1103/PhysRevE.51.131. 
[39] Stauffer D, Aharony A. Introduction to Percolation Theory (Revised 2nd ed.), CRC Press, Boca Raton, 1994.

[40] Tanino Y, Blunt MJ. Capillary trapping in sandstones and carbonates: Dependence on pore structure. Water Resour. Res. 2012;48(8):W08525. Doi:10.1029/2011WR011712.

[41] Wilkinson D, Willemsen JF. Invasion percolation: a new form of percolation theory. Journal of Physics A: Mathematical and General 1983;16(14):3365. doi:10.1088/0305-4470/16/14/028.

[42] Yang Z, Niemi A, Fagerlund F, Illangasekare TH. A generalized approach for estimation of in-plane curvature in invasion percolation models for drainage in fractures. Water Resources Research 2012;48(9):W09507. doi:10.1029/2012WR011829.

[43] Yang Z, Niemi A, Fagerlund F, Hllangasekare TH. Effect of single-fracture aperture statistics on entrapment, dissolution and source depletion behavior of dense nonaqueous phase liquids J. Contam. Hydrol. 2012;133: 1-16. doi:10.1016/j.jconhyd.2012.03.002.

[44] Yang Z, Niemi A, Fagerlund F, Illangasekare TH. Two-phase flow in rough-walled fractures: Comparison of continuum and invasion-percolation models. Water Resour. Res. 2013;49:993-1002. doi:10.1002/wrcr.20111.

[45] Yang Z, Niemi A, Fagerlund F, Illangasekare TH, Detwiler R. Dissolution of dense non-aqueous phase liquids in vertical fractures: effect of finger residuals and dead-end pools, Journal of Contaminant Hydrology 2013;149:88-99. doi:10.1016/j.jconhyd.2013.03.006. 
[46] Ye Z, Liu HH, Jiang Q, Zhou C. Two-phase flow properties of a horizontal fracture: The effect of aperture distribution. Advances in Water Resources 2015;76:43-54. doi:10.1016/j.advwatres.2014.12.001.

[47] Zimmerman RW, Bodvarsson GS. Hydraulic conductivity of rock fractures. Transp. Porous Media 1996;23:1-30. Doi:10.1007/BF00145263. 


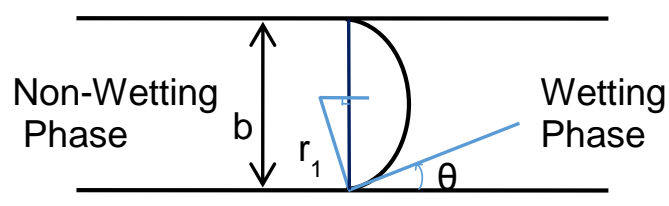

(a)

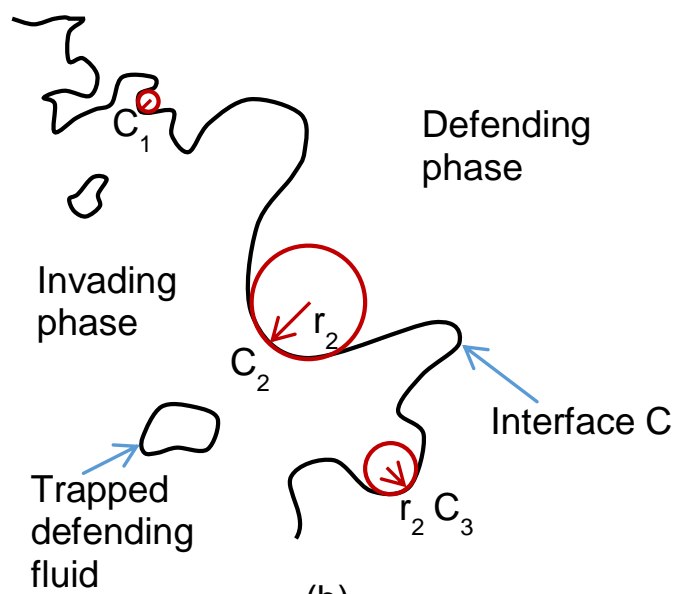

(b)

Fig. 1. Illustration of the two principle radif of curvature for a fluid-fluid interface within a rough-walled fracture: (a) cross section view showing the out-of-plane radius of curvature $r_{1}$ related to the local aperture $b$ and contact angle $\theta$; (b) fracture plane view showing the in-plane radius of curvature $r_{2}$ at points $\mathrm{C}_{1}, \mathrm{C}_{2}$ and $\mathrm{C}_{3}$ on the interface curve C. $\mathrm{C}_{1}, \mathrm{C}_{2}$ and $\mathrm{C}_{3}$ can be determined using osculation circles. In-plane curvature is negative at $C_{1}$ and $C_{2}$, whereas it is positive at $C_{3}$. (Modified after Yang et al. [42] with permission from American Geophysical Union) 


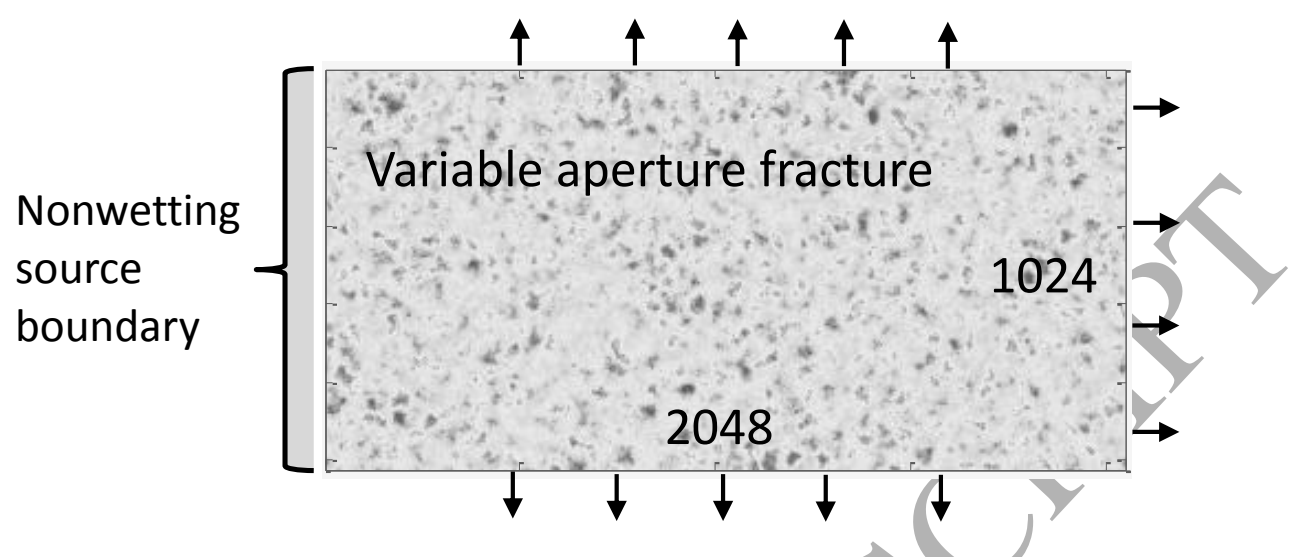

Fig. 2. Schematic of the modeling scenario. The variable aperture fracture is initially fully saturated with the wetting phase. The nonwetting fluid is invading from the left edge, and the wetting fluid is displaced and allowed to leave the fracture through the top, bottom and right edge. 


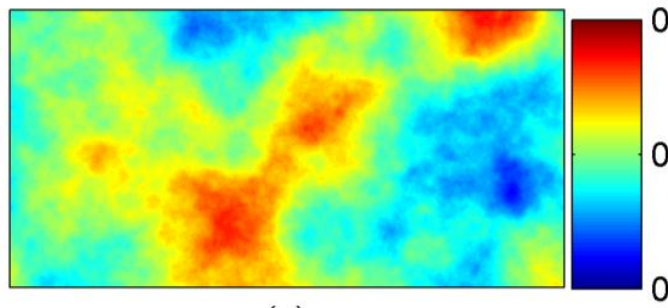

(a)

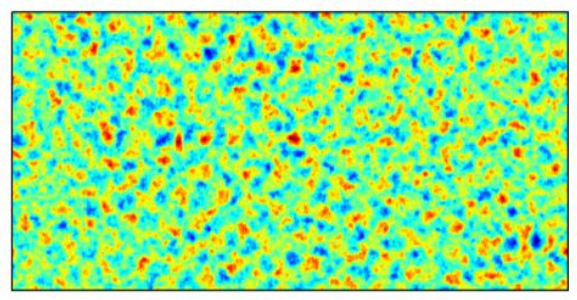

(b)

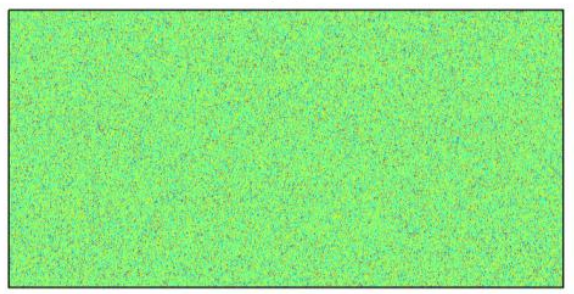

(c)

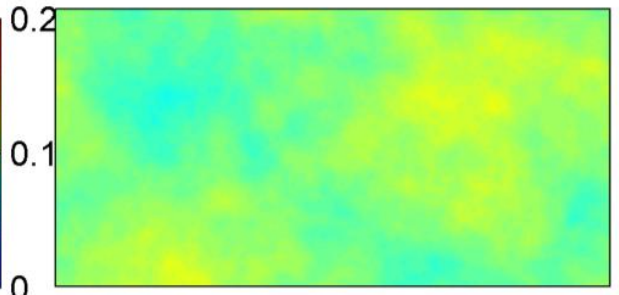

(d)

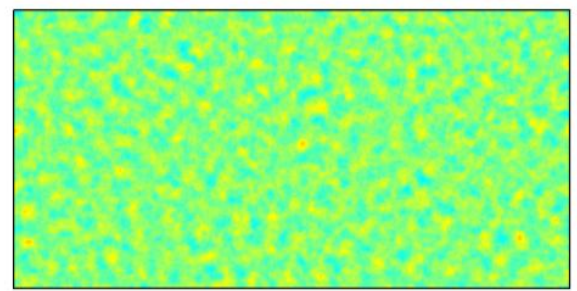

(e)

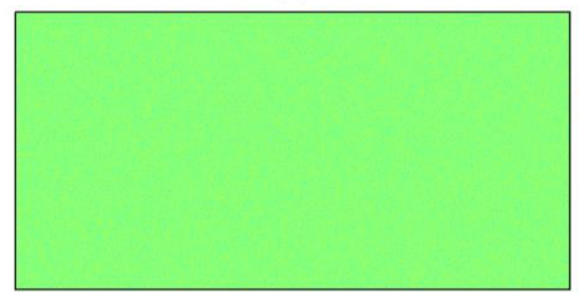

(f)

Fig. 3. Example realizations of the generated aperture field (color bar unit: mm). All realizations have different random seeds. The domain length $L$ is $20.48 \mathrm{~cm}$. (a) Coefficient of variation $\delta=0.25$, mismatch wave number $L / L_{c}=1$; (b) $\delta=0.25, L / L_{c}=16$; (c) $\delta=0.25, L / L_{c}=256$; (d) $\delta=0.0833, L / L_{c}=1$; (e) $\delta=0.0833, L / L_{c}=16$; (f) $\delta=0.0833$, $L / L_{c}=256$ 


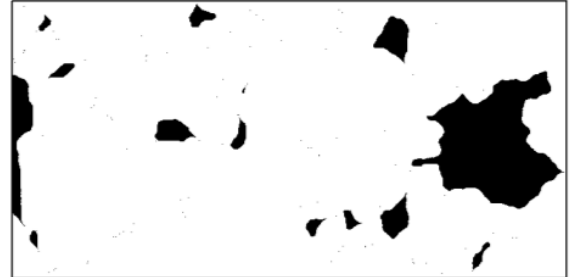

(a)

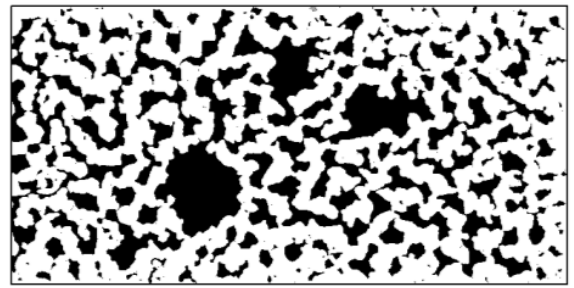

(b)

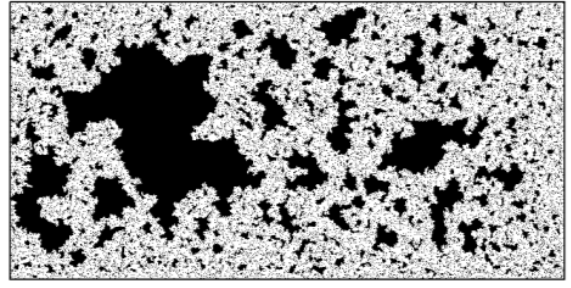

(c)

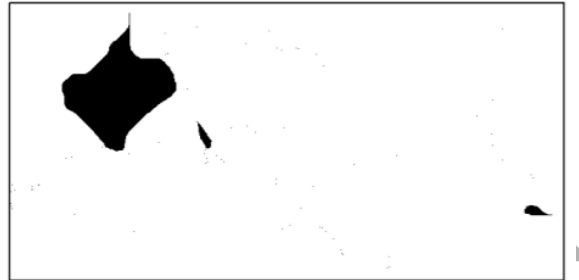

(d)

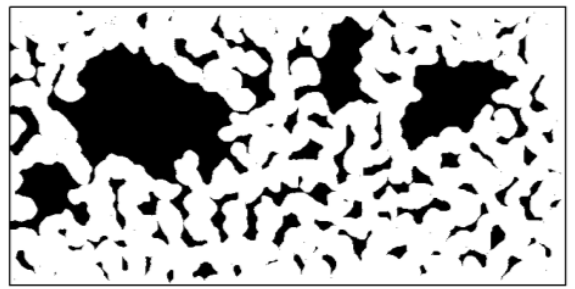

(e)

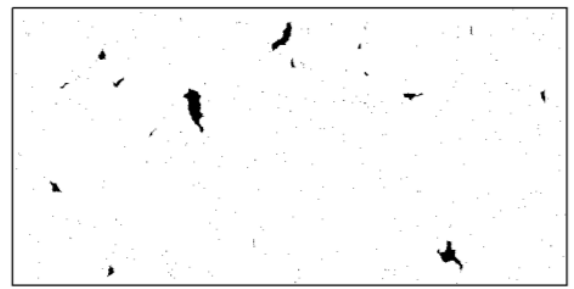

(f)

Fig. 4. Spatial distribution of the trapped wetting fluid (shown in black) for the aperture fields shown in Fig. 3. The domain length $L$ is $20.48 \mathrm{~cm}$. (a) Coefficient of variation $\delta=$ 0.25 , mismatch wave number $L / L_{c}=1$; (b) $\delta=0.25, L / L_{c}=16$; (c) $\delta=0.25, L / L_{c}=256$; (d) $\delta=0.0833, L / L_{c}=1$; (e) $\delta=0.0833, L / L_{c}=16$; (f) $\delta=0.0833, L / L_{c}=256$. 


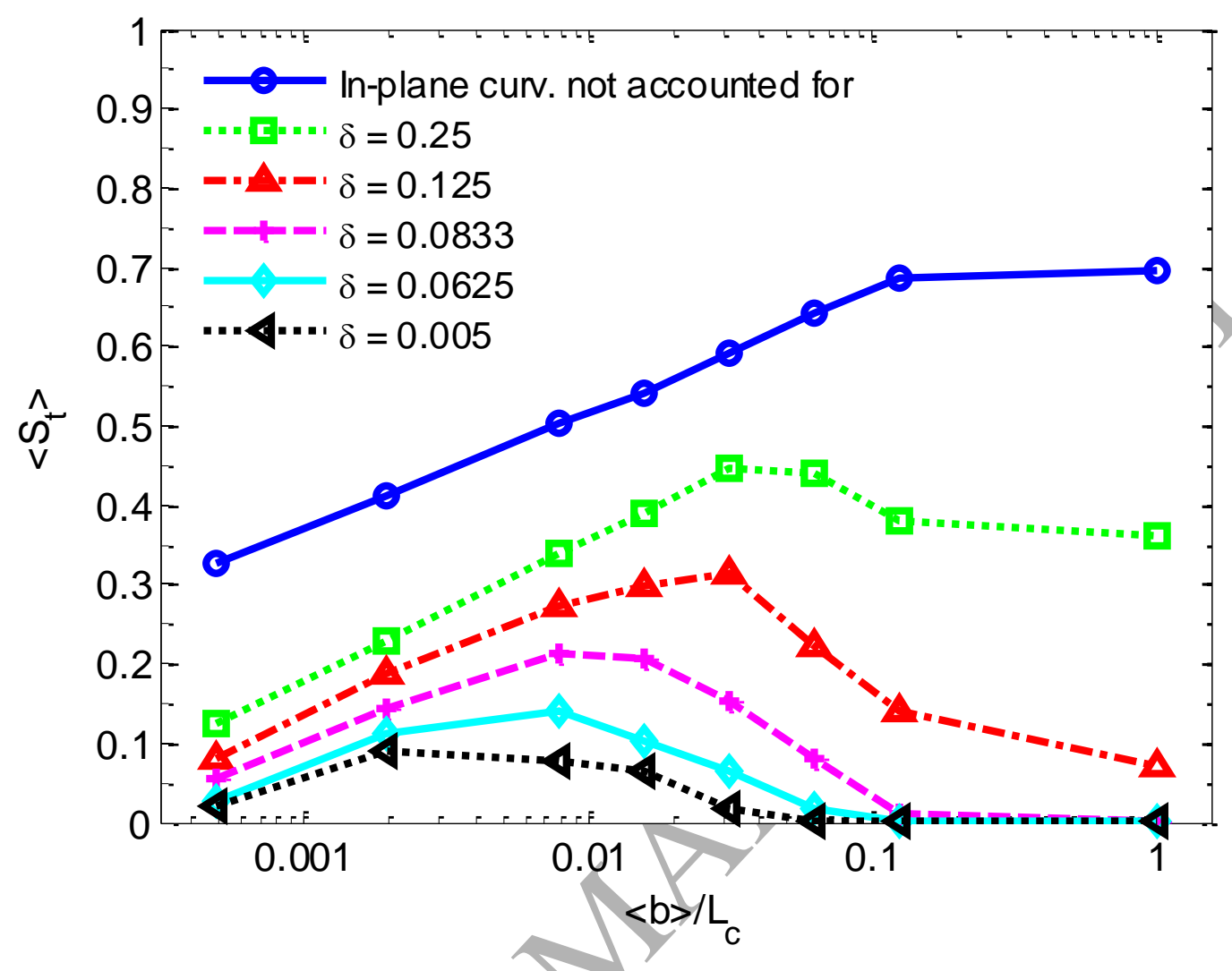

Fig. 5. Average trapped phase saturation $\left\langle S_{t}\right\rangle$ as a function of the aperture correlation scale $L_{c}$ and for different coefficients of variation $\delta$. The IP limit corresponds to the a simulation for which the in-plane curvature has not been accounted for when computing capillary pressures. 

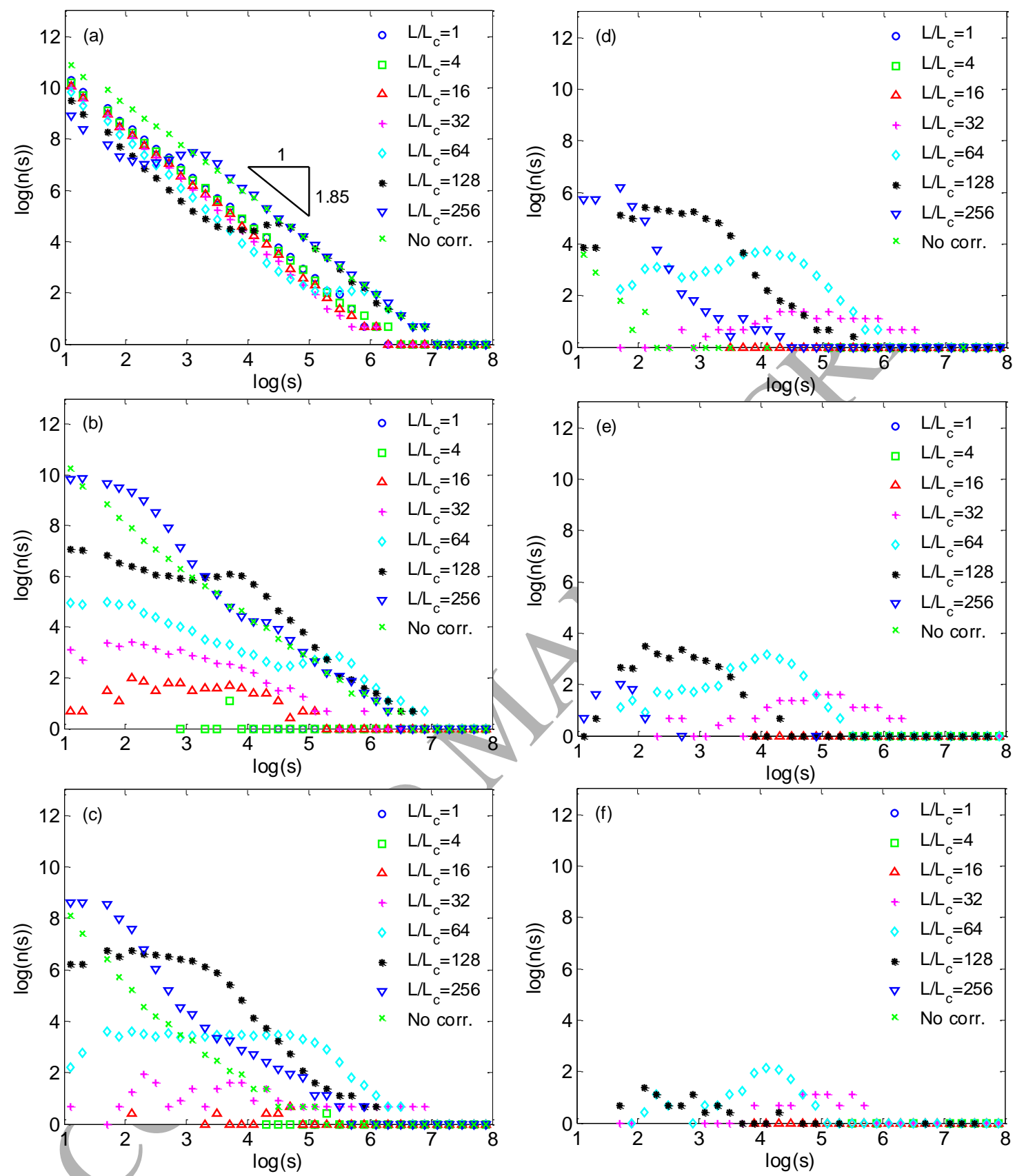

Fig. 6. Trapped fluid cluster size distributions. (a) IP model without the effect of in-plane curvature; (b f) IP modified to include effect of in-plane curvature, coefficient of variation $\delta=0.25,0.125,0.0833,0.0625$ and 0.005 , respectively. 

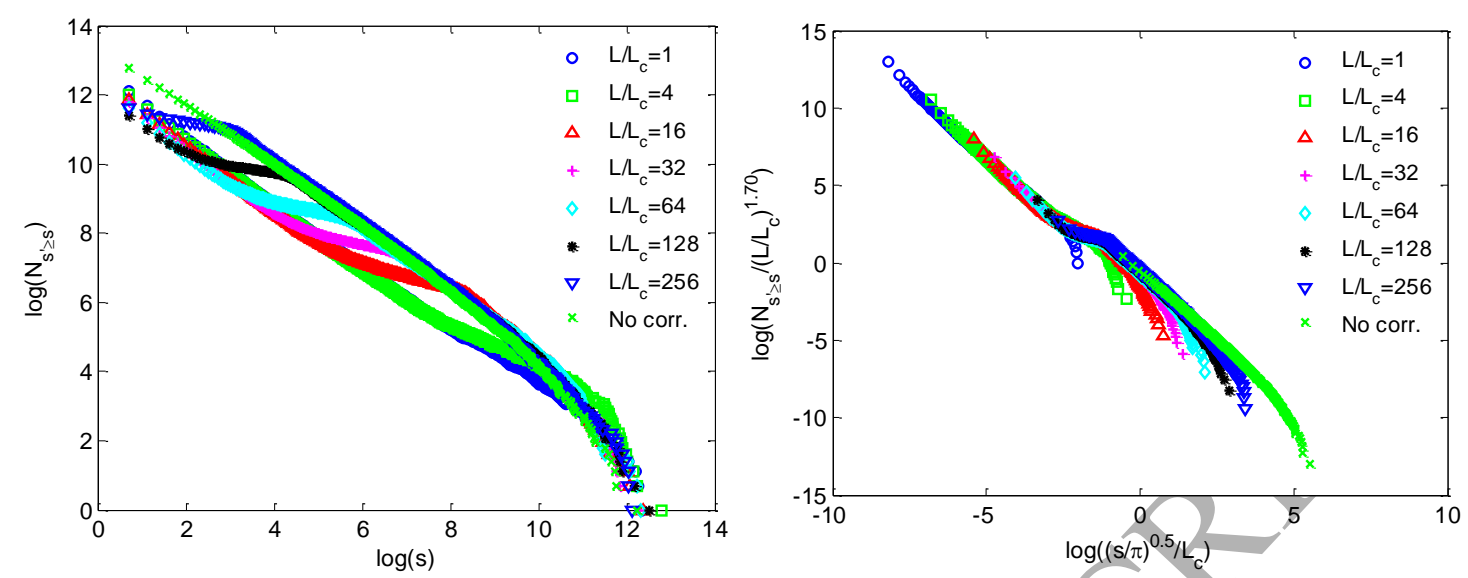

Fig. 7. IP disregarding the effect of in-plane curvature. Left: cumulative cluster size distribution; Right: collapse to a master curve. 

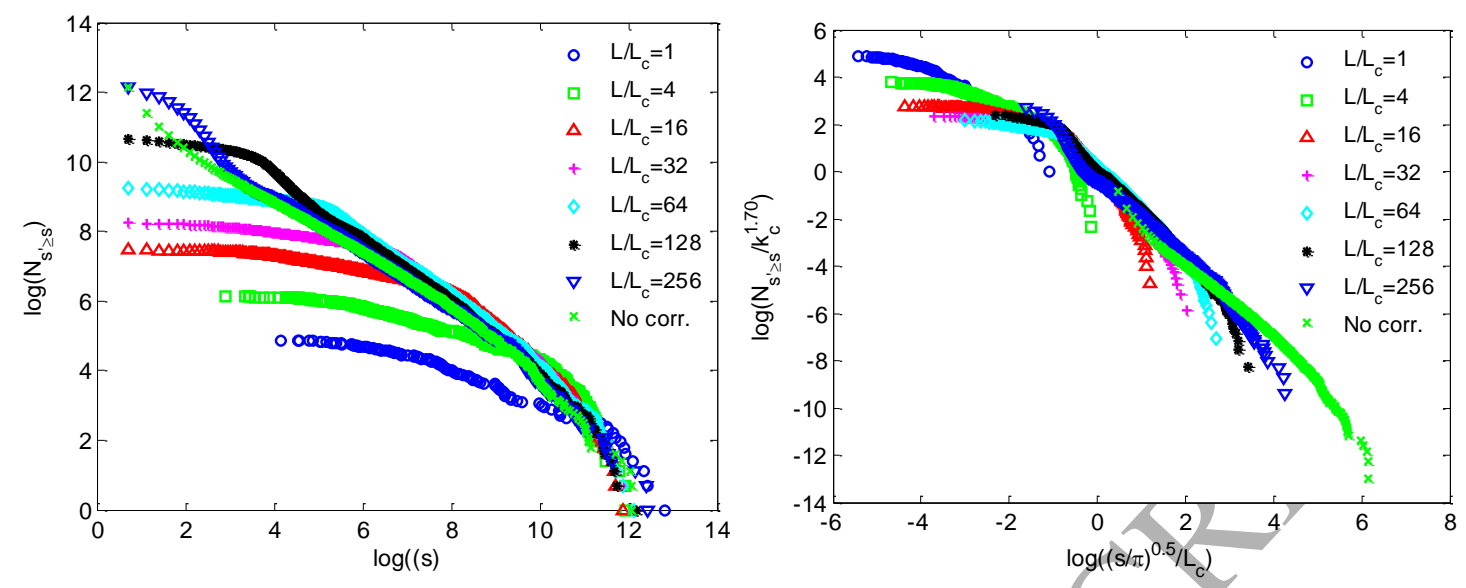

Fig. 8. IP accounting for the effect of in-plane curvature, $\delta=0.25$. Left: cumulative cluster size distribution; Right: collapse of the curves. 


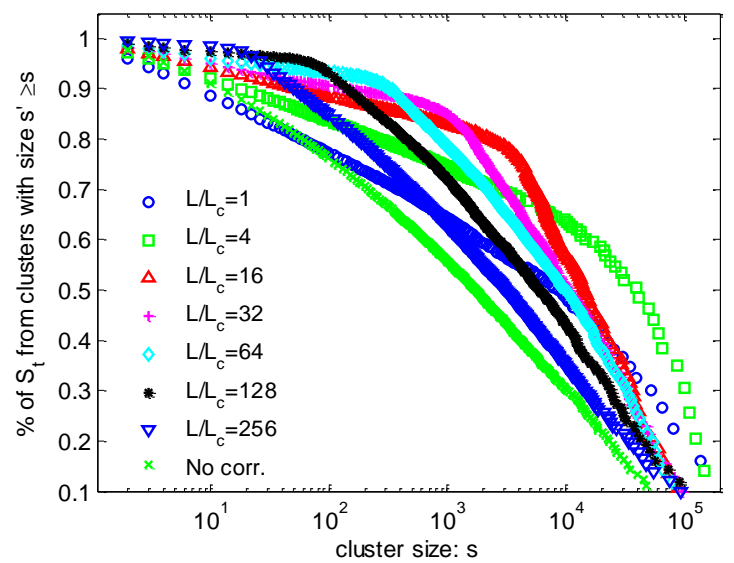

(a)

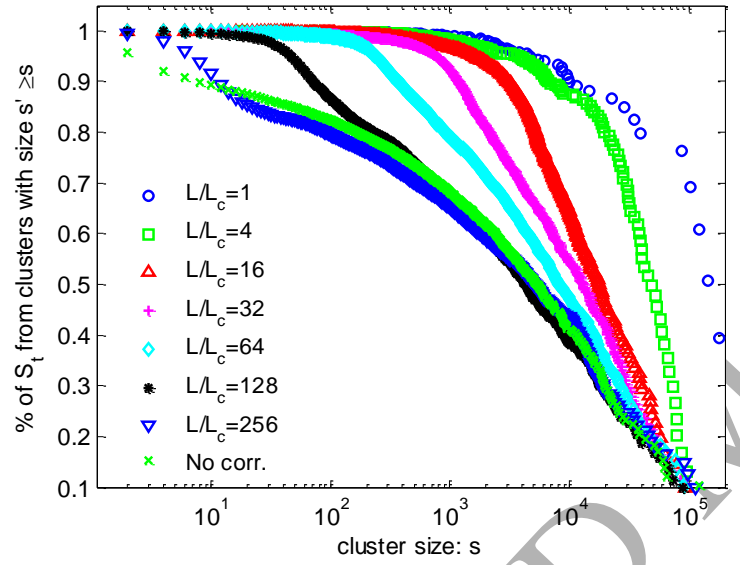

(b)

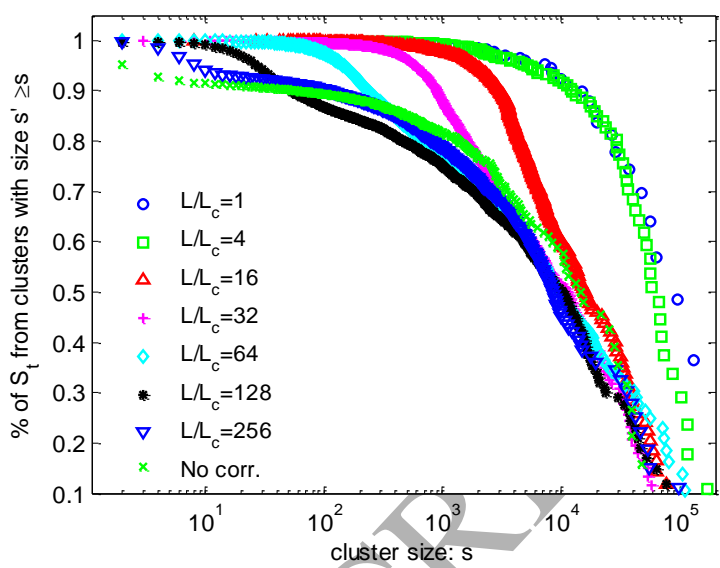

(c)

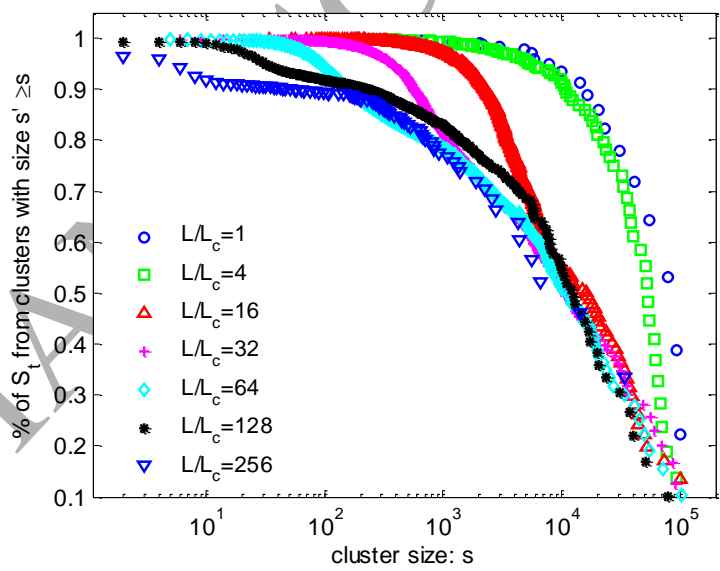

(d)

Fig. 9. Normalized trapped saturation from clusters of sizes larger than or equal to a

certain size $s$. (a) IP model without the effect of in-plane curvature; (b d) IP modified to include the effect of in-plane curvature, coefficient of variation $\delta=0.25,0.125$ and 0.0833 , respectively.

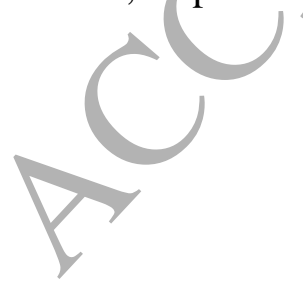




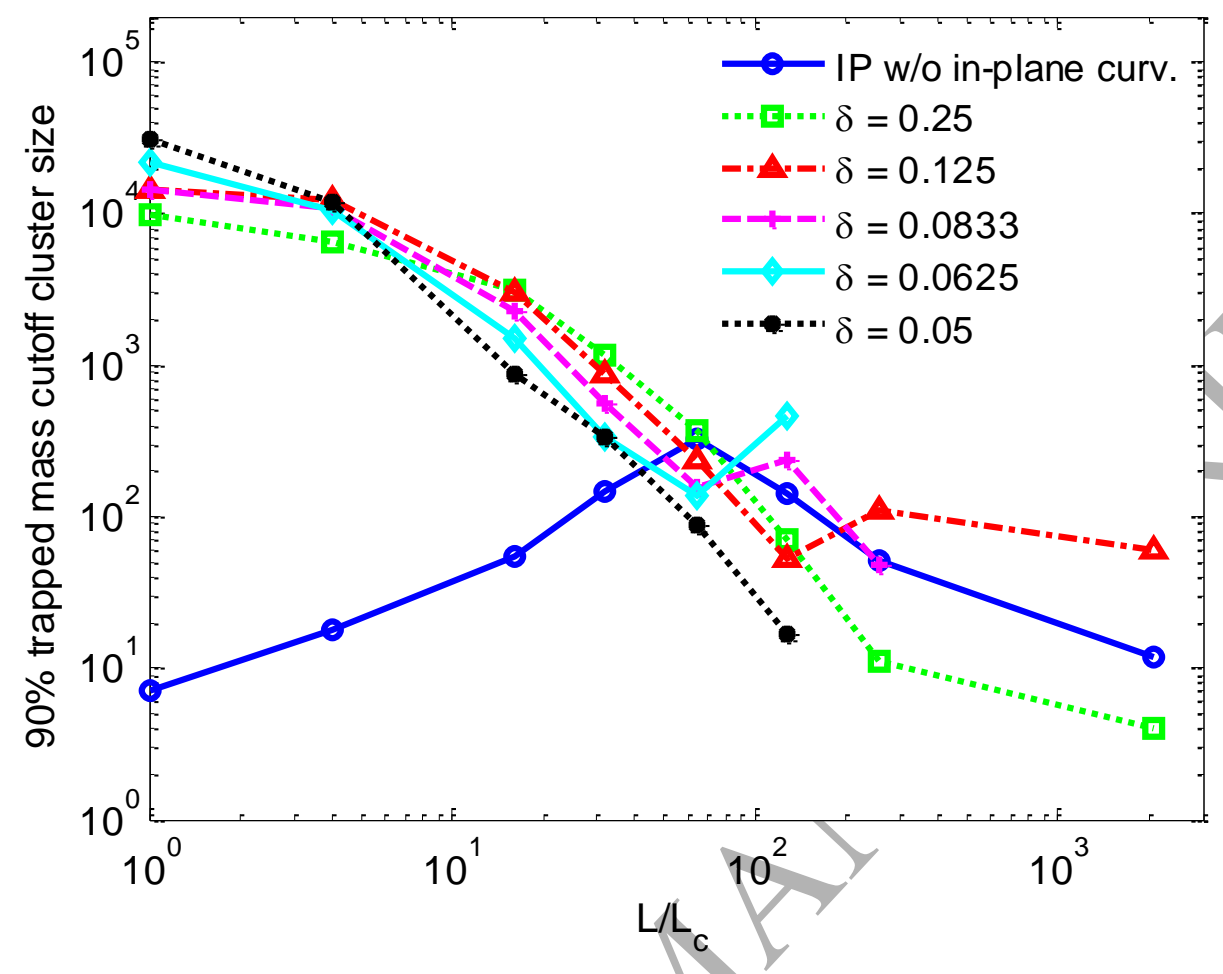

Fig. 10. Cutoff cluster size at $90 \%$ of total trapped mass (from large cluster end). 


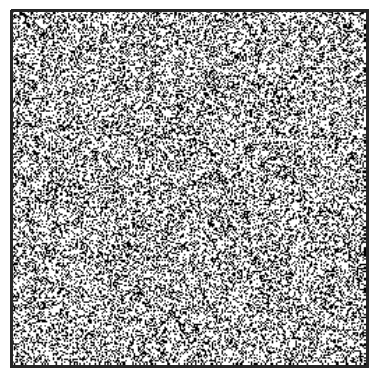

(a)

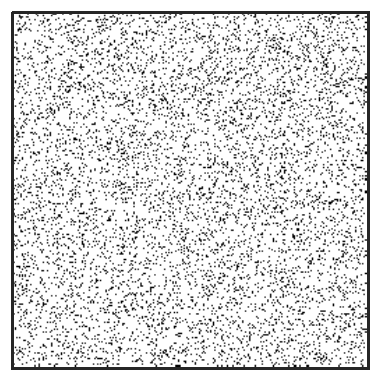

(d)

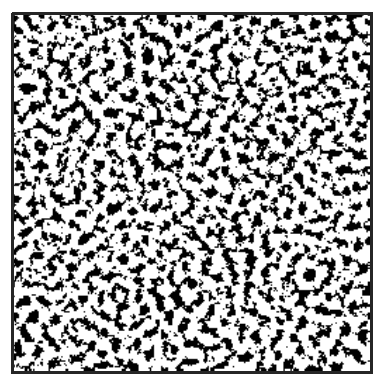

(b)

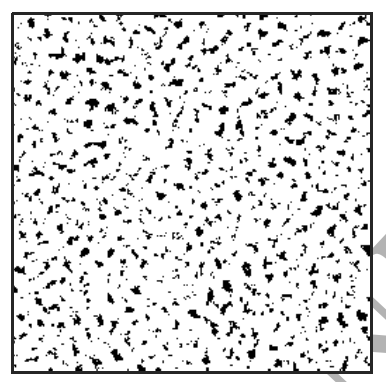

(e)

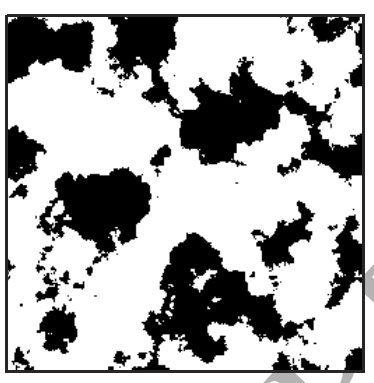

(c)

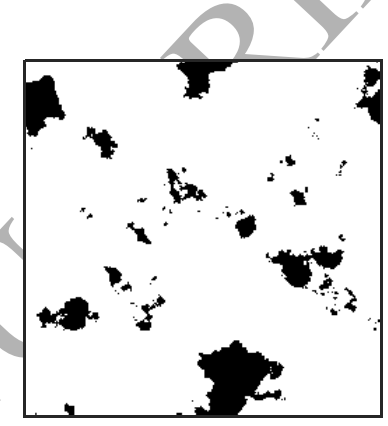

(f)

Fig. 11. Segmented aperture fields with a threshold aperture value of $b_{\mathrm{th}}=0.9\langle b\rangle$, where

$\langle b\rangle$ is the mean aperture. Black are apertures with values below the threshold. (a)

Coefficient of variation $\delta=0.25$, mismatch wave number $L / L_{c}=256$; (b) $\delta=0.25, L / L_{c}=$ 16; (c) $\delta=0.25, L / L_{c}=2$; (d) $\delta=0.0833, L / L_{c}=256$; (e) $\delta=0.0833, L / L_{c}=16$; (f) $\delta=$ $0.0833, L / L_{c}=2$. Note that these fields are generated with a smaller domain length $L$ than that of the aperture field used for simulating fluid displacement. Thus, one should not compare these $L / L_{c}$ values with those in the previous figures. The number of apertures below the threshold is larger in plots (a, b, and c) than in plots (d, e, and f). In plot (a) the configuration of the small apertures is in clusters of different sizes, while in plot (d) the low apertures form isolated small clusters. In the fields (c and f) this effect does not come to play, as structure is determined by the correlation length. 